\title{
ORIGINAL ARTICLE \\ Dietary intake of pantothenic acid is associated with cerebral amyloid burden in patients with cognitive impairment
}

\author{
Jae-Ho Lee', Soo-Yeon Ahn', Hyon Ah Lee², Kyoung Sook Won³, Hyuk Won Chang', \\ Jungsu S. Oh ${ }^{5}$ and Hae Won Kim ${ }^{3 *}$ \\ 'Department of Anatomy, Keimyung University Dongsan Medical Center, Daegu, Republic of Korea; ${ }^{2}$ Department \\ of Neurology, Keimyung University Dongsan Medical Center, Daegu, Republic of Korea; ${ }^{3}$ Department of Nuclear \\ Medicine, Keimyung University Dongsan Medical Center, Daegu, Republic of Korea; ${ }^{4}$ Department of Radiology, \\ Semyung Radiology Clinic, Gumi, Republic of Korea; ${ }^{5}$ Department of Nuclear Medicine, Asan Medical Center, \\ University of Ulsan College of Medicine, Seoul, Republic of Korea
}

\section{Abstract}

Alzheimer's disease (AD) is a neurodegenerative disease characterized by the deposition of amyloid- $\beta$ peptide $(A \beta)$ in diffuse and neuritic plaques. Previous research has suggested that certain vitamins may prevent this process. In the present study, we evaluated the relationship between vitamin intake and cerebral $\mathrm{A} \beta$ burden in patients with cognitive impairment. This study included 19 patients with subjective cognitive impairment and 30 patients with mild cognitive impairment. All patients underwent brain MRI and ${ }^{18}$ F-florbetaben positron emission tomography. The Food Frequency Questionnaire was used to evaluate dietary intake of the 15 vitamins. Intake of vitamin B6 $(p=0.027)$, vitamin $\mathrm{K}(p=0.042)$, vitamin A $(p=0.063)$, riboflavin $(p=0.063), \beta$-carotene $(p=0.081)$, pantothenic acid $(p=0.092)$, and niacin $(p=0.097)$ was higher in the $A \beta$-positive group than in the $A \beta$-negative group. Multivariate linear regression analysis revealed that pantothenic acid intake was an independent determinant of cerebral $\mathrm{A} \beta$ burden $(\beta=0.287$, $p=0.029$ ). No significant correlations were observed between cerebral A $\beta$ burden and the intake of other vitamins. Our findings demonstrated that pantothenic acid intake may be associated with increased cerebral A $\beta$ burden in patients with cognitive impairment. These results may offer insight into potential strategies for AD prevention.

Keywords: Alzheimer's disease; mild cognitive impairment; pantothenic acid; subjective cognitive impairment; vitamin; diet

Received: 29 April 2018; Revised: 25 September 20 8; Accepted: 5 November 20 I8; Published: I0 December 20 I8

T he prevalence and economic costs of Alzheimer's disease (AD) have continued to increase along with increases in the number of older adults in the population (1). Although the diagnosis of $\mathrm{AD}$ is primarily based on clinical symptoms, recent advances in genetics and neuroimaging have indicated that these modalities may aid in the diagnosis of AD (2-4). Additional research has suggested that positron emission tomography (PET) with ${ }^{18} \mathrm{~F}$-florbetaben $\left({ }^{18} \mathrm{~F}-\mathrm{FBB}\right)$ can be used to identify cerebral amyloid- $\beta(A \beta)$ pathology in patients undergoing assessments for $\mathrm{AD}(5,6)$. They showed a sensitivity of $80 \%$ and a specificity of $91 \%$ of PET scan for the diagnosis of Alzheimer'sdisease (6).

Although the etiology of the disease remains to be fully established, evidence suggests that the $A \beta$ peptide plays an important role in the pathogenesis of $\mathrm{AD}(5-7)$. In addition, several risk factors for AD have been identified, including age, certain genetic alleles, and specific nutritional characteristics (8-12). Accumulating evidence indicates that diet - one of the most important modifiable lifestyle factors - may play a role in preventing or delaying cognitive decline and AD (13-16). Epidemiological studies have reported that low intake of vitamins increases the risk of $\mathrm{AD}$, and that several vitamins may be associated with the pathological processes of AD (17-20). However, whether vitamin intake influences the accumulation of cerebral $A \beta$ remains to be investigated.

Previous studies have established the validity of reliability on the Food Frequency Questionnaire (FFQ), which was developed to assess the association between chronic diseases and diet in Korean populations $(21,22)$. In the present study, we aimed to evaluate the association between vitamin intake and cerebral $A \beta$ burden in patients with cognitive impairment using ${ }^{18} \mathrm{~F}$-FBB PET and the 
FFQ. We hope that the results of our study will improve our understanding of $\mathrm{AD}$ pathogenesis, which may aid in the treatment and prevention of $\mathrm{AD}$.

\section{Materials and methods}

\section{Study population}

The present prospective study included consecutive patients (age range: 50-90 years) who had visited the memory clinic at Keimyung University Dongsan Medical Center (Daegu, Korea) for the evaluation of cognitive function between June 2015 and January 2017. All patients underwent standard clinical and neuropsychological evaluations. All patients were divided into syndromal categories based on the 2018 National Institute on Aging-Alzheimer's Association Research Framework: subjective cognitive impairment (SCI) and mild cognitive impairment (MCI) (23). The Mini-Mental State Examination (MMSE), Digit Span Memory Test, Korean-Boston Naming Test (K-BNT), Rey-Osterrieth Complex Figure Test, and Recognition Trial (RCFT) were used to assess cognitive function (24). SCI was defined as subjective memory disorder wherein patients report worsening of their thinking abilities, including memory, but the decline cannot be verified by the tests. MCI was defined as a condition in which subjects had mild but measurable changes in thinking abilities that are noticeable to the person affected and to family members and friends, but do not affect the individual's ability to carry out everyday activities (2). All participants underwent FFQ, brain magnetic resonance imaging, and ${ }^{18} \mathrm{~F}$-FBB PET within 4 weeks of visiting the clinic. Patients with AD or an MMSE score $<20$ were excluded to ensure the reliability of the FFQ. Patients with conditions that could affect cognition (e.g. vascular dementia, a history of psychiatric episodes or substance abuse, or a previous diagnosis of dementia) were also excluded. And patients with other neurodegenerative diseases (e.g. Parkinson's disease); inflammatory brain diseases (multiple sclerosis); medications history such as sedatives, tranquilizers, and anticholinergics; and cancers were also excluded. The study was approved by the Institutional Review Board of Dongsan Medical Center, and written informed consent was obtained from all participants or caregivers.

\section{Dietary assessment}

The categories listed in the FFQ were based on questions from the 2005 Korean Health and Nutrition Survey. A well-trained dietary interviewer used the FFQ to record participants' typical dietary intake. The questionnaire consisted of a list of foods with standard serving sizes, for which patients were requested to select from among nine frequency categories: three times daily, twice daily, once daily, five or six times weekly, three or four times weekly, once or twice weekly, two or three times monthly, once monthly, and never or seldom. Portion sizes were classified into three categories (i.e. small, medium, and large) based on the patient's intake in relation to an appropriately defined unit (e.g. cup or bowl). Dietary intake of nutrients and food groups was assessed using computerized assessment software (CAN-Pro 4.0, Korean Nutrition Society, Seoul, Korea).

\section{Amyloid PET}

A PET/CT system (Biograph mCT-64, Siemens Healthcare, Knoxville, TN) was used to acquire threedimensional ${ }^{18} \mathrm{~F}$-FBB PET images $90-100 \mathrm{~min}$ after the intravenous injection of ${ }^{18} \mathrm{~F}-\mathrm{FBB}(300 \mathrm{MBq})$. Nonenhanced, low-dose CT was performed for attenuation correction and localization. A light, foam-rubber holder was used for fixation of the head. The PET images were subjected to iterative reconstruction using ordered subset expectation maximization. Attenuation correction of PET images was performed using attenuation data from the CT images.

Quantitative analyses were conducted on volumes of interest (VOI) using PMOD software (PMOD Technologies Ltd., Zurich, Switzerland), as previously described (5). Image processing was performed using SPM12 (Wellcome Department of Imaging Neuroscience, Institute of Neurology, University College London) implemented in MATLAB 2013a (MathWorks Inc., MA, USA) and MRIcro version 1.37 (Chris Rorden, Columbia, SC, USA, www.mricro.com).

Each MRI and PET image was co-registered using a standard mutual information algorithm and spatially normalized. An automated anatomical labeling template was subsequently applied for standardized sampling of count densities in VOIs. VOIs were individually defined in the bilateral frontal, temporal, and parietal cortices; anterior and posterior cingulate; and cerebellar cortex. Standardized uptake values were obtained from the regional VOIs, and regional standardized uptake value ratios (SUVRs) were calculated by dividing the standardized uptake values for the different target regions by that for the reference region (i.e. cerebellar cortex). A composite SUVR was calculated as the mean of the values for the frontal, parietal, lateral, temporal, and occipital cortices as well as the anterior and posterior cingulate, as previously described (6). Regional and composite SUVRs were used to evaluate the relationship between vitamin intake and cerebral $\mathrm{A} \beta$ burden. Patients with a composite SUVR $\geq 1.5$, which is considered indicative of an abnormally high cerebral $\mathrm{A} \beta$ burden, were considered positive for $\mathrm{A} \beta$ ( $\mathrm{A} \beta$-positive group), while those with a composite SUVR $<1.5$ were considered negative for $\mathrm{A} \beta$ (A $\beta$-negative group) (6). We then compared differences in vitamin intake between the $A \beta$-positive and $A \beta$-negative groups. This analysis was performed in blinded manner. 


\section{Statistical analyses}

All statistical analyses were performed using IBM SPSS statistics for Windows, V.20 (IBM Corp). Mann-Whitney U tests, Kruskal-Wallis tests, and simple correlation analyses were used to analyze associations between variables. We also assessed the relationship between vitamin intake, MMSE score, and SUVR (cerebral A $\beta$ burden) using Pearson's correlation coefficients and multiple linear regression analyses adjusted for age, sex, and body mass index (BMI). APOE4 as a covariate in the primary model was excluded to avoid variance inflation given its high correlation with cerebral A $\beta$ burden $(25,26)$. A $P$-value of $<0.05$ was considered statistically significant. Data for all study variables are expressed as means \pm standard deviations.

\section{Results}

A total of 49 patients with cognitive impairment were enrolled in the present study. Among them, 19 patients were clinically diagnosed with SCI, while the remaining 30 were diagnosed with MCI. The demographic, clinical, and biochemical characteristics of the SCI and MCI groups are shown in Table 1. MMSE scores were significantly lower in patients with MCI than in those with SCI $(p<0.001)$. Increased cerebral $\mathrm{A} \beta$ burden was observed in $10.5 \%(2 / 19)$ of patients with SCI and $23.3 \%(7 / 30)$ of patients with MCI, although there was no significant difference between the groups ( $p=0.247)$. A negative correlation between cerebral $\mathrm{A} \beta$ burden and MMSE score was shown $(r=-0.395, p=0.007)$.

Vitamin intake for the $A \beta$-negative and $A \beta$-positive groups is presented in Table 2. Intake of vitamin $\mathrm{K}$ $(p=0.042)$ and vitamin B6 $(p=0.027)$ was significantly higher in the $A \beta$-positive group than in the $A \beta$-negative group. Similar tendencies were observed for vitamin A $(p=0.063), \beta$-carotene $(p=0.081)$, riboflavin $(p=0.063)$, niacin $(p=0.097)$, and pantothenic acid $(p=0.092)$, although these results did not reach statistical significance. When stratifying patients into SCI and MCI, a correlation between cerebral $\mathrm{A} \beta$ burden and intake of pantothenic acid was found not in SCI $(r=-0.126, p=0.618)$ but in MCI $(r=0.494, p=0.008)$.

Pearson's correlation analysis revealed that the intake of pantothenic acid was positively correlated with composite $\operatorname{SUVR}(r=0.303, p=0.041$; Fig. 1$)$. When stratified by SUVR, this correlation was found not in $A \beta$-negative group ( $r=0.034, p=0.844$ ) but in $\mathrm{A} \beta$-positive group $(r=0.789, p=0.012)$. No additional associations were observed between vitamin intake and composite SUVR. The results of the multiple linear regression analysis adjusted for age, sex, and BMI are presented in Table 3 . Intake of pantothenic acid was identified as an independent determinant of composite $\operatorname{SUVR}\left(R^{2}=0.289, p=0.029\right)$.
Table 1. Characteristics of patients ${ }^{\mathrm{a}}$

\begin{tabular}{lccc}
\hline & \multicolumn{1}{c}{$\mathrm{SCl}$} & \multicolumn{1}{c}{$\mathrm{MCl}$} & P value \\
\cline { 2 - 3 } & $(\mathrm{n}=19)$ & $(\mathrm{n}=30)$ & \\
\hline Age, $y$ & $62.7(5.5)$ & $64.3(9.7)$ & 0.540 \\
Female, \% & 46.4 & 53.6 & 0.763 \\
BMI & $24.3(4.4)$ & $23.6(2.1)$ & 0.479 \\
Education,y & $14.33(3.5)$ & $12.8(5.6)$ & 0.310 \\
APOE4+, \% & 21.8 & 24.3 & 0.610 \\
SUVR for A $\beta$ & $1.26(0.12)$ & $1.35(0.23)$ & 0.140 \\
MMSE & $28.9(1.2)$ & $25.7(2.6)$ & $<0.00 \mathrm{I}$ \\
Digit span & $11.7(2.3)$ & $10.0(2.2)$ & $<0.00 \mathrm{I}$ \\
K-BNT & $51.8(3.9)$ & $46.3(9.4)$ & $<0.00 \mathrm{I}$ \\
RCFT & $33.0(2.4)$ & $30.6(5.3)$ & $<0.00 \mathrm{I}$ \\
\hline
\end{tabular}

${ }^{a}$ All values are means (SD). K-BNT: Korean-Boston Naming Test; RCFT: Rey-Osterrieth Complex Figure Test and Recognition Trial.

Table 2. Comparisons of vitamin intake between $A \beta$ negative and $\mathrm{A} \beta$ positive groups ${ }^{\mathrm{a}}$

\begin{tabular}{|c|c|c|c|}
\hline & $\begin{array}{c}A \beta \text { negative } \\
(\mathrm{n}=37)\end{array}$ & $\begin{array}{c}A \beta \text { positive } \\
(n=9)\end{array}$ & $P$ value \\
\hline Vitamin A (ug) & $1392.2(1|6| .0)$ & $1966.4(1143.8)$ & .063 \\
\hline Retinol (ug) & I82.3 (167.8) & I89.9 (79.2) & .207 \\
\hline$\beta$-carotene (ug) & $6949.5(5962.8)$ & $10 \mid 44.4(6721.2)$ & .081 \\
\hline Vitamin D (ug) & $2.8(2.4)$ & $2.6(1.7)$ & 1.00 \\
\hline Vitamin E (mg) & $6.9(8.4)$ & $7.5(2.9)$ & .109 \\
\hline Vitamin K (ug) & $36.5(30.0)$ & $54.7(29.7)$ & .042 \\
\hline Vitamin C (mg) & 267.1 (272.3) & $333.4(172.3)$ & .116 \\
\hline Thyamine (mg) & $2.8(2.7)$ & $3.4(1.5)$ & .097 \\
\hline Riboflavin (mg) & $2.5(2.2)$ & $3.0(1.0)$ & .063 \\
\hline Niacin (mg) & $19.9(16.6)$ & $24.6(9.9)$ & .097 \\
\hline Vitamin B6 (mg) & $0.5(0.5)$ & $0.6(0.1)$ & .027 \\
\hline Folic acid (ug) & $223.8(197.6)$ & $249.2(121.8)$ & .286 \\
\hline Vitamin BI2 (ug) & $3.0(4.4)$ & $3.3(2.3)$ & .273 \\
\hline Pantothenic acid (mg) & I.2(0.9) & $1.6(0.9)$ & .092 \\
\hline Biotin (ug) & $6.5(3.8)$ & $7.9(5.6)$ & .605 \\
\hline
\end{tabular}

${ }^{\text {a } A l l ~ v a l u e s ~ a r e ~ m e a n s ~(S D) . ~}$

No such independent relationships were observed between composite SUVR and other vitamins.

We then divided patients into two groups based on median pantothenic acid intake $(1.27 \mathrm{mg})$, following which we compared regional cerebral $\mathrm{A} \beta$ burden between patients with high and low pantothenic acid intake (Table 4). In all cerebral regions, regional SUVR was significantly higher in the high-intake group than in the low-intake group. Multivariate analyses adjusted for age, sex, and BMI revealed that regional SUVR in the frontal, parietal, and temporal cortices was associated with pantothenic acid intake (Table 5). Although a similar tendency was observed in the cingulate cortices, this result did not reach statistical significance (right, $p=0.073$ and left, $p=0.063$ ). 


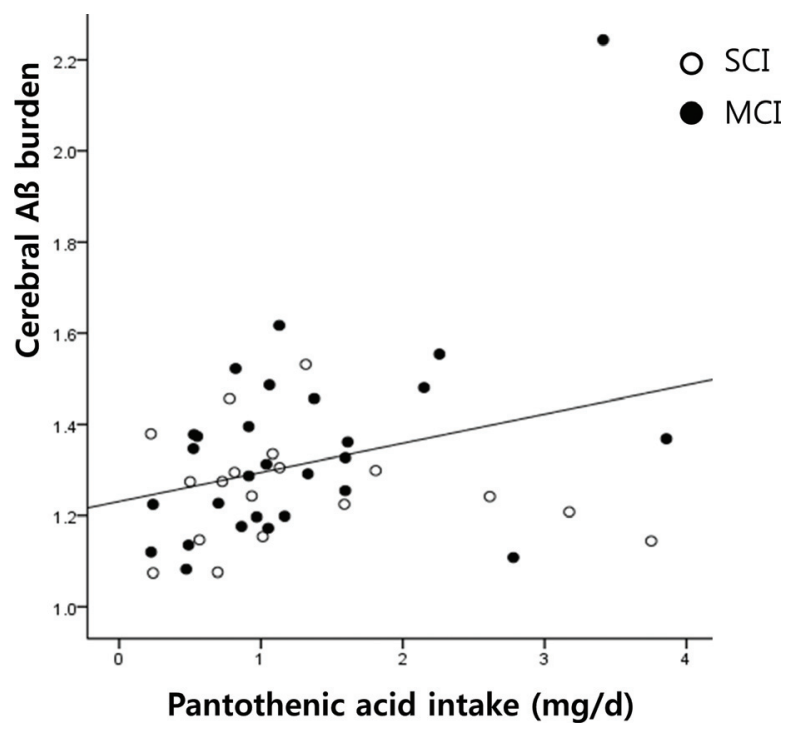

Fig. 1. Positive correlation between pantothenic acid intake and cerebral $\beta$-amyloid burden in SCI and MCI.

Table 3. The relationships of cerebral $\beta$-amyloid burden with vitamin intake

\begin{tabular}{lccc}
\hline & Adjusted $R^{2}$ & Standardized $\beta$ & $P$ value \\
\hline Vitamin A & - & .115 & .398 \\
Retinol & - & .073 & .593 \\
$\beta$-carotene & - & .103 & .450 \\
Vitamin D & - & .100 & .456 \\
Vitamin E & - & .079 & .564 \\
Vitamin K & - & .140 & .306 \\
Vitamin C & - & -.010 & .942 \\
Thyamine & - & .104 & .443 \\
Riboflavin & - & .103 & .451 \\
Niacin & - & .108 & .429 \\
Vitamin B6 & - & .133 & .333 \\
Folic acid & - & .126 & .362 \\
Vitamin B12 & - & .132 & .326 \\
Pantothenic acid & - & .287 & .029 \\
Biotin & - & .096 & .487 \\
\hline
\end{tabular}

\section{Discussion}

In the present study, we aimed to evaluate the relationship between vitamin intake and cerebral $\mathrm{A} \beta$ burden in patients with cognitive impairment. Some of SCI population and patients with MCI had $\mathrm{A} \beta$ positive result. Although subjects with $\mathrm{A} \beta$ positive are not cognitively impaired, these would be changed into dementia within 20 years. Our results indicated that cerebral $A \beta$ burden was positively associated with dietary intake of pantothenic acid in patients with MCI and SCI. Interestingly, subjects with $\mathrm{A} \beta$ positive showed this correlation with more significance. Furthermore, higher intake of pantothenic acid
Table 4. Comparison of regional SUVR between pantothenic acid low-intake and high-intake groups ${ }^{\mathrm{a}}$

\begin{tabular}{lcccc}
\hline \multirow{2}{*}{ Region } & Side & \multicolumn{2}{c}{ Pantothenic acid } & P value \\
\cline { 3 - 4 } & & $\begin{array}{c}\text { Low-intake } \\
(\mathrm{n}=19)\end{array}$ & $\begin{array}{c}\text { High-intake } \\
(\mathrm{n}=30)\end{array}$ \\
\hline Frontal lobe & $\mathrm{Rt}$ & $\mathrm{I} .15(0.1 \mathrm{I})$ & $\mathrm{I} .29(0.2 \mathrm{I})$ & $\mathbf{0 . 0 I}$ \\
& $\mathrm{Lt}$ & $\mathrm{I} .17(0.12)$ & $\mathrm{I} .32(0.2 \mathrm{I})$ & $\mathbf{0 . 0 \mathrm { I }}$ \\
Parietal lobe & $\mathrm{Rt}$ & $\mathrm{I} .17(0.14)$ & $\mathrm{I} .29(0.29)$ & $\mathbf{0 . 0 6}$ \\
& $\mathrm{Lt}$ & $\mathrm{I} .25(0.13)$ & $\mathrm{I} .39(0.32)$ & $\mathbf{0 . 0 4}$ \\
Temporal lobe & $\mathrm{Rt}$ & $\mathrm{I} .22(0.12)$ & $\mathrm{I} .33(0.19)$ & $\mathbf{0 . 0 2}$ \\
& $\mathrm{Lt}$ & $\mathrm{I} .3 \mathrm{I}(0.12)$ & $\mathrm{I} .43(0.24)$ & $\mathbf{0 . 0 3}$ \\
Cingulate & $\mathrm{Rt}$ & $\mathrm{I} .56(0.16)$ & $\mathrm{I} .72(0.27)$ & $\mathbf{0 . 0 2}$ \\
& $\mathrm{Lt}$ & $\mathrm{I} .44(0.17)$ & $\mathrm{I} .60(0.35)$ & $\mathbf{0 . 0 5}$ \\
\hline
\end{tabular}

${ }^{a}$ All values are mean Fazekas rating.

Table 5. The relationships of regional cerebral $\beta$-amyloid burden with intake of pantothenic acid

\begin{tabular}{lcccc}
\hline Region & Side & Adjusted $R^{2}$ & Standardized $\beta$ & $P$ value \\
\hline Frontal lobe & $\mathrm{Rt}$ & 0.279 & 0.272 & $\mathbf{0 . 0 3 9}$ \\
& $\mathrm{Lt}$ & 0.305 & 0.321 & $\mathbf{0 . 0 1 4}$ \\
Parietal lobe & $\mathrm{Rt}$ & 0.194 & 0.309 & $\mathbf{0 . 0 3 0}$ \\
& $\mathrm{Lt}$ & 0.266 & 0.267 & $\mathbf{0 . 0 4 4}$ \\
Temporal lobe & $\mathrm{Rt}$ & 0.297 & 0.295 & $\mathbf{0 . 0 2 4}$ \\
& $\mathrm{Lt}$ & 0.396 & 0.339 & $\mathbf{0 . 0 0 6}$ \\
Cingulate & $\mathrm{Rt}$ & - & 0.248 & 0.073 \\
& $\mathrm{Lt}$ & - & 0.242 & 0.063 \\
\hline
\end{tabular}

from food sources was linearly associated with cerebral $\mathrm{A} \beta$ burden in various brain regions, even after controlling for multiple covariates.

In general, humans require adequate amounts of four fat-soluble vitamins (A, D, E, K) and nine water-soluble vitamins, which comprise vitamin $\mathrm{C}$ and the eight $\mathrm{B}$ vitamins: thiamine $\left(\mathrm{B}_{1}\right)$, riboflavin $\left(\mathrm{B}_{2}\right)$, niacin $\left(\mathrm{B}_{3}\right)$, pantothenic acid $\left(B_{5}\right)$, vitamin $B_{6}$, vitamin $B_{7}$, folate $\left(B_{9}\right)$, and vitamin $B_{12}(17,18)$. Pantothenic acid $\left(B_{5}\right)$ is a substrate for the synthesis of the ubiquitous coenzyme $\mathrm{A}(\mathrm{CoA})$ (27). Beyond its role in oxidative metabolism, CoA contributes to the structure and function of brain cells via its involvement in the synthesis of cholesterol, amino acids, phospholipids, and fatty acids $(28,29)$. Notably, pantothenic acid is also involved in the synthesis of multiple neurotransmitters and steroid hormones via pathways involving CoA (28).

Supplementation of several vitamins such as folate, vitamin $\mathrm{B}_{12}$, and vitamin $\mathrm{B}_{6}$ has been recommended for the prevention of cognitive decline (14-19). In contrast, our results indicate that increased pantothenic acid intake may increase cerebral A $\beta$ burden. And over intakes of Vitamin $\mathrm{K}$ and Vitamin B6 tended to be risk factor for $\mathrm{A} \beta$ burden uptake. Vitamin $\mathrm{K}$ and Vitamin $\mathrm{B} 6$ tended to 
be risk for $A \beta$ burden uptake. However, multivariate analysis showed that only pantothenic acid is a strong factor to increase cerebral $\mathrm{A} \beta$ burden.

Major food sources of pantothenic acid include chicken, beef, potatoes, oat cereals, tomatoes, eggs, broccoli, and whole grains (29). Previous study showed an average pantothenic acid intake $(4.5 \mathrm{mg} /$ day for men and $4.0 \mathrm{mg} /$ day for women), which is higher than our result (27). As previously stated, total intake of pantothenic acid may be low because of its widespread occurrence in food. Although these foods are well-known sources of neuroprotection $(30,31)$, excessive intake may induce unexpected effects on CoA pathways, proteins, and carbohydrate synthesis. Pantothenic acid, a precursor to CoA, plays an indispensable role in carboxylic acid and fatty acid metabolism in various organisms (32). Therefore, increased CoA activity via high pantothenic acid intake may induce inappropriate consumption of biological defense resources such as pro-inflammatory cytokines, adhesion molecules, and acute response proteins associated with inflammation. However, further studies are required to verify this hypothesis.

The present study possesses several limitations of note. First, the FFQ is limited in assessing absolute intake because it is based on a finite list of food items, categories, and intake levels. And the intake of other compounds and health supplements also influence the cerebral $A \beta$ burden though they were included in FFQ. Therefore, evaluation using the FFQ should be followed by analysis of nutrient levels in the blood. In addition, because all study participants resided in South Korea, we were unable to examine the influence of regional differences in environment, socioeconomic status, and health-related habits. Thus, further prospective studies involving various populations are required. Despite these limitations, the present study is advantageous in that a novel cross-sectional approach was used to investigate the association between dietary intake of pantothenic acid and cerebral $\mathrm{A} \beta$ burden.

\section{Conclusion}

The present study is the first to demonstrate a positive association between cerebral $A \beta$ burden and dietary intake of pantothenic acid in patients with cognitive impairment using PET and the FFQ. Notably, this association was observed in most cerebral regions and following multivariate analysis, suggesting that pantothenic acid intake influences cerebral $A \beta$ burden. Further studies are required to confirm our findings and to elucidate the molecular mechanisms underlying this association.

\section{Acknowledgements}

This work was supported by a National Research Foundation of Korea (NRF) grant funded by the Korea Government (MSIP) (no. 2014R1A5A2010008) and grant from the Korea Healthcare Technology R\&D Project through the Korea Healthy Industry Development, funded by Ministry of Health and Welfare, Republic of Korea (HI15C3102).

\section{Conflict of interest and funding}

The authors have not received any funding or benefits from industry or elsewhere to conduct this study.

\section{References}

1. Hebert LE, Weuve J, Scherr PA, Evans DA. Alzheimer disease in the United States (2010-2050) estimated using the 2010 census. Neurology 2013; 80: 1778-83. doi: 10.1212/WNL.0b013e $31828726 f 5$.

2. McKhann GM, Knopman DS, Chertkow H, Hyman BT, Jack CR Jr, Kawas CH, et al. The diagnosis of dementia due to Alzheimer's disease: recommendations from the National Institute on Aging-Alzheimer's Association workgroups on diagnostic guidelines for Alzheimer's disease. Alzheimers Dement 2011; 7: 263-9. doi: 10.1016/j.jalz.2011.03.005.

3. Brickman AM, Provenzano FA, Muraskin J, Manly JJ, Blum S, Apa Z, et al. Regional white matter hyperintensity volume, not hippocampal atrophy, predicts incident Alzheimer disease in the community. Arch Neurol 2012; 69: 1621-7. doi: 10.1001/ archneurol.2012.1527.

4. Ryan NS, Keihaninejad S, Shakespeare TJ, Lehmann M, Crutch SJ, Malone IB, et al. Magnetic resonance imaging evidence for presymptomatic change in thalamus and caudate in familial Alzheimer's disease. Brain 2013; 136: 1399-1414. doi: 10.1093/ brain/awt065.

5. Mikolajczyk K, Szabatin M, Rudnicki P, Grodzki M, Burger C. A JAVA environment for medical image data analysis: initial application for brain PET quantitation. Med Inform (Lond) 1998; 23: 207-14.

6. Barthel H, Gertz HJ, Dresel S, Peters O, Bartenstein P, Buerger $\mathrm{K}$, et al. Cerebral amyloid-beta PET with florbetaben (18F) in patients with Alzheimer's disease and healthy controls: a multicentre phase 2 diagnostic study. Lancet Neurol 2011; 10: 424-35. doi: 10.1016/S1474-4422(11)70077-1.

7. Hardy JA, Higgins GA. Alzheimer's disease: the amyloid cascade hypothesis. Science 1992; 256: 184-5.

8. Yaffe K, Kanaya A, Lindquist K, Simonsick EM, Harris T, Shorr RI, et al. The metabolic syndrome, inflammation, and risk of cognitive decline. JAMA 2004; 292: 2237-42.

9. Rosengren A, Skoog I, Gustafson D, Wilhelmsen L. Body mass index, other cardiovascular risk factors, and hospitalization for dementia. Arch Intern Med 2005; 165: 321-6.

10. Jack CR Jr, Lowe VJ, Weigand SD, Wiste HJ, Senjem ML, Knopman DS, et al. Serial PiB and MRI in normal, mild cognitive impairment and Alzheimer's disease: implications for sequence of pathological events in Alzheimer's disease. Brain 2009; 132: 1355-65. doi: 10.1093/brain/awp062.

11. van Norden AG, van Dijk EJ, de Laat KF, Scheltens P, Olderikkert MG, de Leeuw FE. Dementia: Alzheimer pathology and vascular factors: from mutually exclusive to interaction. Biochem Biophys Acta 2012; 1822: 340-9. doi: 10.1016/j. bbadis.2011.07.003.

12. Latta $\mathrm{CH}$, Brothers HM, Wilcock DM. Neuroinflammation in Alzheimer's disease; A source of heterogeneity and target for personalized therapy. Neuroscience 2015; 302: 103-111. doi: 10.1016/j.neuroscience.2014.09.061. 
13. Morris MC, Evans DA, Bienias JL, Tangney CC, Bennett DA, Aggarwal N, et al. Dietary intake of antioxidant nutrients and the risk of incident Alzheimer disease in a biracial community study. JAMA 2002; 287: 3230-7.

14. Ousset PJ, Nourhashemi F, Reynish E, Vellas B. Nutritional status is associated with disease progression in very mild Alzheimer disease. Alzheimer Dis Assoc Disord 2008; 22: 66-71. doi: 10.1097/WAD.0b013e31815a9dbb.

15. Kamphuis PJ, Scheltens P. Can nutrients prevent or delay onset of Alzheimer's disease? J Alzheimers Dis 2010; 20: 765-75. doi: 10.3233/JAD-2010-091558.

16. Gu Y, Scarmeas N. Dietary patterns in Alzheimer's disease and cognitive aging. Curr Alzheimer Res 2011; 8: 510-19.

17. Paleologos M, Cumming RG, Lazarus R. Cohort study of vitamin C intake and cognitive impairment. Am J Epidemiol 1998; 148: 45-50.

18. Masaki KH, Losonczy KG, Izmirlian G, Foley DJ, Ross GW, Petrovitch $\mathrm{H}$ et al. Association of vitamin $\mathrm{E}$ and $\mathrm{C}$ supplement use with cognitive function and dementia in elderly men. Neurology 2000; 54: 1265-72.

19. Luchsinger JA, Tang MX, Shea S, Mayeux R. Antioxidant vitamin intake and risk of Alzheimer disease. Arch Neurol 2003; 6: 203-8.

20. Zandi PP, Anthony JC, Khachaturian AS, Stone SV, Gustafson D, Tschanz JT, et al. Cache County Study Group. Reduced risk of Alzheimer disease in users of antioxidant vitamin supplements: the Cache County Study. Arch Neurol 2004; 61: 82-8. doi: 10.1001/archneur.61.1.82

21. Kim WY, Yang EJ. A study on development and validation of food frequency questionnaire for Koreans. Korean J Nutr 1998; 31: $220-30$.

22. Ahn Y, Kwon E, Shim JE, Park MK, Joo Y, Kimm K et al. Validation and reproducibility of food frequency questionnaire for Korean genome epidemiologic study. Eur J Clin Nutr 2007; 61: 1435-41. doi: 10.1038/sj.ejen.1602657

23. Jack CR Jr, Bennett DA, Blennow K, Carrillo MC, Dunn B, Haeberlein SB et al. NIA-AA research framework: toward a biological definition of Alzheimer's disease. Alzheimers Dement 2018; 14: 535-62. doi: 10.1016/j.jalz.2018.02.018.

24. Folstein MF, Folstein SE, McHugh PR. 'Mini-mental state'. A practical method for grading the cognitive state of patients for the clinician. J Psychiatr Res 1975; 12: 189-98.
25. Morris JC, Roe CM, Xiong C, Fagan AM, Goate AM, Holtzman DM et al. APOE predicts amyloid-beta but not tau Alzheimer pathology in cognitively normal aging. Ann Neurol 2010; 67: 122-31. doi: 10.1002/ana.21843.

26. Vidoni ED, Townley RA, Honea RA, Burns JM. Alzheimer disease biomarkers are associated with body mass index. Neurology 2011; 77: 1913-20. doi: 10.1212/ WNL.0b013e318238eec1.

27. Jung S, Kim MK, Choi BY. The long-term relationship between dietary pantothenic acid (vitamin B5) intake and C-reactive protein concentration in adults aged 40 years and older. Nutr Metab Cardiovasc Dis 2017; 27: 806-16. doi: 10.1016/j. numecd.2017.05.008.

28. Slyshenkov VS, Dymkowska D, Wojtczak L. Pantothenic acid and pantothenol increase biosynthesis of glutathione by boosting cell energetics. FEBS Lett 2004; 569(1e3): 169e72. doi: 10.1016/j.febslet.2004.05.044

29. Zempleni J, Rucker RB, McCormick DB, Suttie JW. Handbook of vitamins, 4th ed. Boca Raton, FL: CRC Press; 2007, p. 240.

30. Friedland RP, Tedesco JM, Wilson AC, Atwood CS, Smith MA, Perry G, et al. Antibodies to potato virus Y bind the amyloid beta peptide: immunohistochemical and NMR studies. J Biol Chem 2008; 283: 22550-6. doi: 10.1074/jbc. M802088200.

31. Park SK, Ha JS, Kim JM, Kang JY, Lee du S, Guo TJ et al. Antiamnesic effect of broccoli (Brassica oleracea var. italica) leaves on amyloid beta $(\mathrm{a} \bigotimes) 1-42$ - induced learning and memory impairment. J Agric Food Chem 2016; 64: 3353-61. doi: 10.1021/acs.jafc.6b00559.

32. Leonardi R, Zhang YM, Rock CO, Jackowski S. Coenzyme A. Back in action. Prog Lipid Res 2005; 44(2e3): 125e53. doi: 10.1016/j.plipres.2005.04.001

\section{*Hae Won Kim}

Department of Nuclear Medicine

Keimyung University School of Medicine

2800 Dalgubeoldaero, Dalseo-Gu

Daegu, 4260I, Republic of Korea

Email: hwkim.nm@gmail.com 